\section{ANOTACIONES SOBRE LOS JUICIOS POR TERRENOS BALDÍOS EN CHIAPAS A PARTIR DE LOS INFORMES DEL JUZGADO (1851-1869) ${ }^{1}$}

Jesús Antonio Cosamalón Aguilar PONTIFICIA UNIVERSIDAD CATÓLICA DEL PERÚ

\begin{abstract}
RESUMEN
Este trabajo pretende, a partir del análisis de algunos documentos provenientes de los juzgados de Chiapas, delimitar la importancia de los juicios por terrenos baldíos en el Estado antes de la época de Porfirio Díaz. El interés fundamental se concentra en establecer que las leyes acerca de los terrenos baldíos probablemente ocasionaron un temprano proceso de acumulación. En ese sentido, la principal contribución consiste en ofrecer datos estadísticos y cualitativos que permiten la elaboración de hipótesis de trabajo coherentes para comprender mejor la evolución de la propiedad agraria en ese período. Por esta razón ofrecemos a los interesados un anexo estadístico con los datos más relevantes de esta documentación, con la esperanza de que pueda guiar futuras investigaciones.
\end{abstract}

\title{
INTRODUCCIÓN
}

Este trabajo pretende, a partir de algunos expedientes ubicados en el Archivo General de la Nación, delimitar las características de los juicios por terrenos baldíos en Chiapas. Nos interesa plantear los problemas que la documentación ofrece y busca construir una perspectiva de investigación coherente para ser puesta a prueba posteriormente. El material que ubicamos ofrece una variada información, pero ésta por sí sola no basta para discutir lo que otros autores, con mayor tiempo de investigación dedicado al tema, han presentado en diversos trabajos. 
Los expedientes hacen posible la presentación de los datos por medio de estadísticas, las cuales ayudan a delimitar los problemas que plantea la documentación. Estos cuadros y gráficos no hacen otra cosa que mostrar, de manera mucho más clara, lo que de otra forma podría quedar confuso. Como ocurre frecuentemente, no demuestran directamente nada, sino grafican aspectos de la realidad que pueden servir posteriormente para establecer hipótesis de investigación.

La exposición la divido en tres partes. En la primera, intento plantear de manera muy general el problema del origen de las haciendas en México y Chiapas. En la segunda, hago un rápido balance de las leyes agrarias que afectaron a la nación y al estado de Chiapas durante el siglo XIX. En la tercera y última parte elaboro una breve crítica documental y presento los datos extraídos de los expedientes.

\section{EL ORIGEN DE LAS HACIENDAS}

El tema de la aparición de la hacienda en América Latina motivó una serie de debates, especialmente atractivos en la década de 1970. Hoy el tema aparece menos frecuentemente en la agenda de los especialistas, a pesar de que no se puede afirmar que todo esté dicho. En los años 70 el tema atrajo numerosas investigaciones, tal vez una de las razones más importantes para ello fue la de tratar de establecer los conceptos adecuados aplicables a la realidad americana a partir de categorías europeas como feudalismo, capitalismo y sus variantes. Es frecuente encontrar que, en esa época, se hablaba de los "pre": pre-capitalismo y pre-industrial, por ejemplo. Hoy se habla del "post": post-industrial y post-moderno. A pesar de esta variación lingüística, que no siempre refleja una mejor comprensión de los procesos históricos, todavía hay muchos aspectos que nos resultan desconocidos en cuanto a la formación de las haciendas.

Las primeras afirmaciones vinculaban de manera demasiado esquemática, tal vez mecánica, la aparición de la hacienda con la despoblación indígena y las encomiendas. Desde esta perspectiva, la grave disminución demográfica, el abuso de los españoles y la anuencia de las

\footnotetext{
${ }^{1}$ Este trabajo fue presentado en el seminario que dicta Juan Pedro Viqueira en El Colegio de México. Agradezco al profesor los comentarios y criticas que han ayudado a mejorar el trabajo. Las deficiencias que aún pueda contener este ensayo son de mi absoluta responsabilidad.
} 
autoridades coloniales, ocasionaron la aparición de haciendas en el territorio hispanoamericano. Así, desde el siglo XVII en México, según Borah, la "economía mexicana estaría ya organizada sobre la base de latifundios y en el peonaje por deudas, rasgos gemelos de la vida mexicana que (...) contribuyeron a provocar la revolución de 1910-1917” (Mörner , 1975: 15-48). Además, desde este punto de vista, no se trata sólo de la posesión de grandes porciones de tierra, rasgo excesivamente legal, sino de la forma de producción de la hacienda: orientada hacia el mercado local y el uso de mano de obra principalmente bajo formas no asalariadas. De esta manera la realidad agraria de México del siglo XX tendría su origen directo en la situación colonial.

Esta tesis ha sido discutida en muchos aspectos. En primer lugar, la encomienda no siempre derivó en hacienda, dada las restricciones legales que la corona aplicó en América especialmente a partir de las Leyes Nuevas de 1542. En segundo lugar, la evidencia muestra que, paradójicamente, la "típica hacienda colonial pudo haber surgido en algunas áreas sólo después de la Independencia" (Mörner, 1975: 16.) En el caso mexicano el asunto es bastante complejo, dado que en algunas zonas el proceso de concentración de tierras parece que se inició desde el siglo XVII, en otras más tardíamente, pero hay acuerdo en colocar al período del presidente Porfirio Díaz como un momento histórico trascendental en la acumulación de terrenos ( De Vos, 1995, 227:265).

En el caso de Chiapas el poder de las órdenes religiosas, especialmente de los dominicos, se ha destacado como el factor más importante en cuanto a la concentración de tierras durante el período colonial. Desde el siglo XVI, dejando de lado los ideales trazados por fray Bartolomé de Las Casas, la orden dominica se encargó de acumular tierras, influencia y poder hasta su expulsión en 1859. ${ }^{2}$ A partir de una serie de artimañas y artificios legales, los religiosos dominicos, al igual que los mercedarios y jesuitas, se las ingeniaron para poseer haciendas y manejar la producción de la zona. No parece existir demasiada duda respecto a este punto en los autores que trabajan el tema. Aunque existen propietarios civiles en Chiapas, su esfuerzo por acumular tierra no tuvo el éxito que acompañó a los religiosos y frecuentemente chocaban con la

\footnotetext{
${ }^{2}$ Dos trabajos resultan importantes para analizar el caso de las haciendas en Chiapas. A. García de León, Resistencia y utopía. Memorial de agravios y crónica de revueltas y profecías acaecidas en la provincia de Chiapas durante los últimos quinientos años de su historia. México. Ediciones Era, 1985, T.I. y M. H. Ruz, Savia india, floración ladina. Apuntes para una historia de las fincas comitecas (siglos XVIII-XIX). México. Conaculta, 1992.
} 
resistencia de los indígenas (Ruz 1992: 110-111). A pesar de todo este proceso de acumulación de tierras por los religiosos y otros grupos, las comunidades indígenas se las ingeniaron para mantener buena parte de sus tierras durante el período colonial, por lo que tampoco se puede afirmar que las haciendas del siglo XX son herederas directas del despojo de tierras efectuado por la iglesia durante los siglos XVI-XIX (Op. cit.: 279).

\section{LAS LEYES AGRARIAS Y LA FORMACIÓN DE LAS HACIENDAS}

Durante la época republicana bajo un manto de igualdad, de raigambre liberal, se aplicaron diversas disposiciones que - en la práctica - motivaron una grave campaña en contra de las tierras de las comunidades. Aunque es posible —al menos se puede dar el beneficio de la dudaque muchas de estas disposiciones fueran realmente diseñadas bajo la creencia de que producirían una capa de pequeños propietarios, libres e independientes de las corporaciones, el efecto concreto fue la depredación de las cada vez más escasas tierras comunales.

Son dos aspectos los que me interesa destacar en términos generales. En primer lugar, las leyes de desamortización de bienes de corporaciones civiles y religiosas, llamada la Ley de Lerdo (1856) y en segundo lugar las diversas disposiciones acerca de los terrenos baldíos. La Ley de Lerdo tenía como uno de sus objetivos las tierras que pertenecían a las órdenes religiosas, pues la ley los obligó a poner en venta las propiedades no vinculadas directamente a sus funciones. Se discute aún hoy, si es que la ley tenía realmente como objeto despojarlas de sus bienes u obligarlas a venderlos. La realidad es que la negativa de las órdenes a poner en venta sus bienes se utilizó como argumento cuando el gobierno decretó su nacionalización en 1859 (Mejía, 1979: 221). Los efectos que esta disposición trajo en las corporaciones civiles, especialmente en las comunidades, son menos conocidos. Parece que su importancia varió en cuanto a las dinámicas regionales y la situación expansiva o no de las haciendas (Menegus, 1995: 144-189).

La ley pretendía convertir a los campesinos en propietarios individuales, ordenándose el fraccionamiento de los pueblos (Mejía, 1979: 227). Los pueblos poseían el fundo legal, que era el área central establecida para el pueblo durante el período colonial; los propios, terrenos 
reservados para que los pueblos consigan ingresos alquilándolos a terceros; las tierras de repartimiento o común, entregadas a las familias para su subsistencia y los ejidos, terrenos de pastos de libre disposición por parte de los miembros de la comunidad (Knowlton, 1991). La ley de 1856 estableció que los ejidos de los pueblos se consideraban fuera del ámbito de la disposición, aunque en algunos casos se intentó incluirlos de facto (Mejía, 1979: 228). En términos generales, los terrenos de común repartimiento fueron el primer objetivo de las medidas liberales a lo largo del siglo XIX, mientras los ejidos, montes y pastos fueron los últimos reductos de la propiedad comunal (Menegus, 1995: 156 y 160). ${ }^{3}$ Los liberales consideraban que la ausencia de propiedad privada contribuía al atraso y aislamiento de los indios, por esta razón se dispuso que los terrenos de común repartimiento se entregaran, con una serie de ventajas fiscales y administrativas, a quienes las poseían en ese momento. Sólo en caso de negativa de compra de parte de sus ocupantes, previo plazo prudencial, se procedería a venderlos a otros postores (Fraser, 1991: 239).

Se desconoce la magnitud de redistribución de la propiedad que ocasionó este proceso en México. Sólo hay datos parciales y bastante más extensos para el caso de las órdenes religiosas. Para el caso de Chiapas la desamortización parece tener efectos relativamente modestos, a juzgar por las cifras de Jan Bazant. Mientras en el estado de México se otorgaron más de dos mil adjudicaciones, en Chiapas sólo se entregaron nueve; dentro de ellas ninguna sobrepasó los diez mil pesos (Bazant, 1995). Ruz indica que los hacendados, más que ir sobre las propiedades comunales, se dirigieron contra los bienes de las cofradías y de las órdenes religiosas, normalmente mejores tierras que los pobres terrenos de los indígenas (Ruz 1992:142-143). ${ }^{4}$ Aún así, el proceso de creación de nuevos propietarios recibió con esta ley un buen respaldo, especialmente cuando algunos futuros hacendados denunciaron los títulos de las comunidades como provenientes de instituciones religiosas, al mismo tiempo que avanzaban sus cercos de ganado mayor (García de León, 1985, I: 157).

\footnotetext{
${ }^{3}$ Los ejidos fueron considerados propiedad de la nación y por tal razón resguardados de la privatización, véase Fraser, Donald J. "La política de desamortización en las comunidades indígenas, 1856-1872" pp. 219-256, en García Martínez, B. (1991).

${ }^{4}$ Además, puede verse De Vos, J., Vivir en frontera. La experiencia de los indios de Chiapas. México, CIESAS, 1994, p. 164.
} 
Entonces, aunque la Ley Lerdo contribuyó — tal vez contrariamente a sus intereses - a ampliar el acaparamiento de tierras en muchos estados, ${ }^{5}$ su efecto en Chiapas no es del todo claro; aparentemente no resulta un elemento definitivo en la conformación de la gran propiedad chiapaneca del siglo XIX.

Otra parte importante de este proceso son las leyes sobre terrenos baldíos que se dieron a lo largo del siglo XIX, por lo menos en el caso de Chiapas. Al igual que los terrenos comunales, las tierras ociosas o baldías, se consideraron un obstáculo al desarrollo de la nación (González, 1995). El intento de repartir estas tierras comienza en fecha tan temprana como 1754, para 1824 el congreso mexicano expidió una ley de colonización que permitió la compra de tierras que no fueran de comunidad o de religiosos. En 1826 la primera ley agraria del estado de Chiapas aceptó la denuncia de terrenos baldíos por particulares, aunque en 1827 se modificó el alcance de la ley para evitar los conflictos entre las fincas y las comunidades. Se estableció límites a los ejidos, pero - según García de León - estas disposiciones no detuvieron el avance de las fincas, aunque no presenta referencias concretas (1985, I: 149). En 1844 la asamblea departamental de Chiapas reglamentó la venta de terrenos baldíos, disposición en la que prácticamente cualquier terreno cuyo dueño no pudiera demostrar su propiedad sería considerado baldío (De Vos, 1994: 161, 255 256). Estas disposiciones son reforzadas en 1855 y con la Ley Lerdo recibieron un nuevo e inesperado apoyo. En 1863 Benito Juárez estableció una nueva ley a favor de la colonización, procurando mejorar el erario público y ampliar la frontera agrícola (Menegus, 1995: 154; Fraser, 1991: 253). Esta ley tenía ciertas dificultades que obstaculizaban su funcionamiento, por ejemplo, suponía el conocimiento de los denunciantes del terreno que declaraban, lo que hacía poco atractiva la oportunidad para los extranjeros que preferían las tierras ya deslindadas y fraccionadas; además, la ley establecía como límite las 2,500 has. (De Vos, 1995: 229 y 233). Por esta razón se considera que la ley de Juárez fue de aplicación bastante limitada, sólo pudo mejorarse hasta 1875 con la nueva ley de colonización y con la supresión de los ejidos en 1878 (De Vos, 1994: 171).

\footnotetext{
${ }^{5}$ La tesis de Jesús E. Mendoza García muestra los efectos de este proceso en Oaxaca, donde la ley intentó crear un mercado de tierras. Bienes de comunidad: cohesión y autonomía de Santo Domingo Tepenene. Oaxaca durante la segunda mitad del siglo XIX, 1856-1910, (Tesis de Licenciatura), México, INAH, 1996, p. III-IV.
} 
En Chiapas la intervención francesa y la posterior restauración del régimen republicano motivaron una nueva ola de denuncia de baldíos, cuya venta — se dijo - era para ayudar a solventar los gastos de guerra (Ruz, 1992: 147). Una vez restaurado el régimen se trataría de dar títulos a los indios, aunque las autoridades informaban, para 1869, que ninguno de ellos lo había solicitado. Este dato resulta muy importante para este ensayo, dado que los registros que analizaremos muestran que sí existió una importante denuncia de terrenos baldíos antes de 1875, aunque no con los alcances de lo que ocurrió después de 1883.

Finalmente, Porfirio Díaz depuró las dificultades legales y en dos etapas sucesivas, 1883 y 1894, ofreció las mejores ventajas, eliminando incluso el límite a la extensión del denuncio. Esto hizo que durante el porfiriato los terrenos adjudicados correspondan al $90 \%$ del total registrado entre 1821 y 1910 (De Vos, 1995: 242). A pesar de estos datos hay dudas respecto al balance final de todo este proceso, pues algunos sostienen que sólo el 5\% de los pueblos logró conservar sus tierras para 1910, mientras otros elevan este porcentaje a 40\% (Schenk, 1991); en todo caso hay que tener bastante cuidado con las estadísticas agrarias pues la confiabilidad de ellas no siempre es adecuada y nos pueden llevar a la confusión. Los efectos de estas disposiciones en Chiapas son mejor conocidos para el período del porfiriato. Se considera que entre 1877 y 1910 el número de haciendas se duplicó, por ejemplo el $27 \%$ del territorio del estado fue denunciado por compañías privadas (García de León, I: 167 y 173).

¿Por qué la importancia de los terrenos baldíos en Chiapas? Una primera respuesta marca la diferencia con el centro de México. En este último se menciona la falta de tierras disponibles (Mörner, 1975: 28); Menegus considera que la importancia de las leyes de baldíos en el estado de México fue bastante menor (1995: 162). Parece que las posibilidades de terreno considerado ocioso fueron mayores en Chiapas, especialmente porque muchas de las tierras que se podían denunciar como baldías eran en realidad milpas de temporal, cultivadas en las laderas de los montes por los indígenas que las usaban desde mucho tiempo antes. Formaban parte de su economía, pero no había forma de demostrar legalmente su pertenencia. ${ }^{6}$ Esto significa que

\footnotetext{
${ }^{6}$ García de León afirma que el terreno baldío dio origen al peonaje por deudas: "en las laderas y montes cuya conformación hacía imposible el uso del arado, tierras por lo general baldías, se cultivaba maíz a la usanza milenaria de la región... para estas tareas, los frailes emplearon aparceros sujetos al pago de una renta: de ahí que baldío, el trabajador en tierras baldías, se convirtiera en sinónimo de aparcero sujeto por deudas" (García de León, I: 108).
} 
posiblemente muchas de las tierras baldías no fueran tales, en el estricto sentido de la palabra. No eran tierras abandonadas, sino tierras de uso temporal y tradicional de las comunidades.

Resumiendo este punto se puede notar que las disposiciones sobre terrenos baldíos, básicamente después de la segunda mitad del XIX, son las que posiblemente producen el mayor acaparamiento de tierras en Chiapas. Una precisión que tal vez se pueda agregar es que se debe tener en cuenta de manera más profunda el efecto de las leyes de baldíos anteriores al porfiriato. Aunque varios autores insisten — con razón— que antes de 1875 hay un proceso de acumulación de tierras bajo las leyes de baldíos, no he encontrado referencias sistemáticas al proceso. Las estadísticas que voy a presentar a continuación intentan ayudar a eliminar esa deficiencia, mostrando algunos datos tomados de los registros judiciales que ratifican la afirmación de los especialistas.

\section{LAS ESTADÍSTICAS DE LOS JUICIOS CIVILES DE CHIAPAS $(1851,1852,1868$ Y 1869)}

\section{LOS DOCUMENTOS: DIFICULTADES Y APORTES}

Antes de pasar a presentar los datos concretos correspondientes a Chiapas, es necesario dar algunas breves indicaciones sobre el origen de la documentación. ${ }^{7}$ Los expedientes que ubicamos en el Archivo General de la Nación (AGN), corresponden a informes de los tribunales solicitados por las autoridades a cada corte de justicia de los distintos estados, acerca de los diversos juicios civiles, criminales, decomisos y causas administrativas que se ventilaban en cada juzgado. En dos secciones del AGN (Justicia y Corte Suprema de Justicia), se conservan numerosos expedientes remitidos por los juzgados que informan lo solicitado por las autoridades. Lamentablemente,

\footnotetext{
${ }^{7}$ Todos los cuadros y gráficos están basados en la combinación de los siguientes documentos del Archivo General de la Nación: "Estado que manifiesta los expedientes civiles de hacienda que se giran por este juzgado con expresión de reos, delitos, estado de ellos y fecha del último trámite" (1851), Justicia, Leg. 389, Exp. 3, f.22-25; "Estado Nº 1 y 4 Causas civiles de hacienda" (1852), Justicia, Leg. 389, Exp. 21, f.222-226; "Estado que manifiesta los negocios que han cursado en el último semestre del año próximo pasado" (1868), Corte Suprema de Justicia, Caja 77, Exp. 101 y "Tribunal de circuito de Yucatán, Campeche, Tabasco y Chiapas, Juzgado de Distrito del Estado Libre y Soberano de
} 
como cualquier pesquisa lo puede notar, son bastante más numerosos para otros estados de México en comparación a Chiapas. Además, otro problema evidente es que no siempre los documentos manejan el mismo formato de información. En algunos casos, sobre todo en 1851 y 1852, se presentan bastantes causas administrativas dentro de las listas de juicios, las mismas que no son mencionadas en 1868 y 1869. También el periodo que cubre cada documento es distinto, los dos iniciales abarcan un año, pero los dos últimos sólo cubren un semestre. A pesar de ello parece coherente incluirlos en el análisis, dado que los informes son acerca de los juicios en trámite, de manera que de todas formas nos dan un indicador acerca del impacto de las disposiciones. Por otro lado, esta es la documentación con que contamos y mal que bien puede mostrarnos una imagen, aunque sea parcial, de los juicios llevados en el Estado. Indudablemente esta información debe ser complementada con otros documentos relativos al tema y desde este punto de vista resulta insuficiente. No es mi intención pretender que con esta documentación se puede demostrar suficientemente la interpretación que propongo, pero mi propuesta consiste en señalar que los juicios por terrenos baldíos deben ser considerados seriamente en el estudio del proceso de la conformación de la propiedad agraria en Chiapas. En ese sentido quiero mostrar que el estudio de esta documentación por medio de métodos cuantitativos puede ayudarnos a esclarecer y perfilar mejor el proceso, aunque, evidentemente, esto no debe suplantar el registro y análisis de la imprescindible documentación regional.

Los expedientes consignan, en general, la causa del juicio, el nombre de los denunciantes o implicados, la fecha de inicio del proceso, su estado actual, el lugar del juicio, la fecha del último trámite, la causa de su paralización, si ese es el caso, y en el documento de 1868, lo cual resulta de sumo interés, el nombre y ubicación del terreno baldío denunciado. Al ubicar los expedientes preferimos dedicar nuestro esfuerzo - absolutamente inicial - a contextualizar las causas civiles, a pesar de la existencia de otros juicios criminales, administrativos o por defraudación a derechos estatales. Los juicios civiles mostraban en abundancia la presencia de denuncias por terrenos baldíos, lo que causó nuestro interés en el tema, en desmedro de los demás aspectos. Una indicación final al respecto es que en algunos casos hemos tratado de combinar la 
información, especialmente sobretodo en el caso de los nombres, por que debido a nuestro escaso conocimiento sobre los apellidos de la zona no siempre se alcanzó a determinarlos con exactitud. De manera que el análisis de la documentación que presento está fundamentado en los expedientes ya mencionados, combinándolos entre sí para tener una información más clara. El resultado lo muestro en los anexos Núm. 1 y Num. 2, los cuales indican:

1.- Aquellas personas que están citadas más de una vez como encausados o denunciantes.

2.- La lista de las personas, fechas, lugar del juicio, nombre de la finca, ubicación de ella, estado del juicio y fecha del último trámite.

Estos anexos, que son una imagen parcial de los documentos, tienen el objeto de mostrar a los interesados el tipo de información disponible en el archivo, la cual esperamos facilite el trabajo más profundo en el tema.

LOS JUICIOS Y LA INFORMACIÓN QUE NOS PROPORCIONAN

En primer lugar veamos el número de causas civiles presentadas por año, la cual se refleja en el gráfico Núm. $1{ }^{8}$

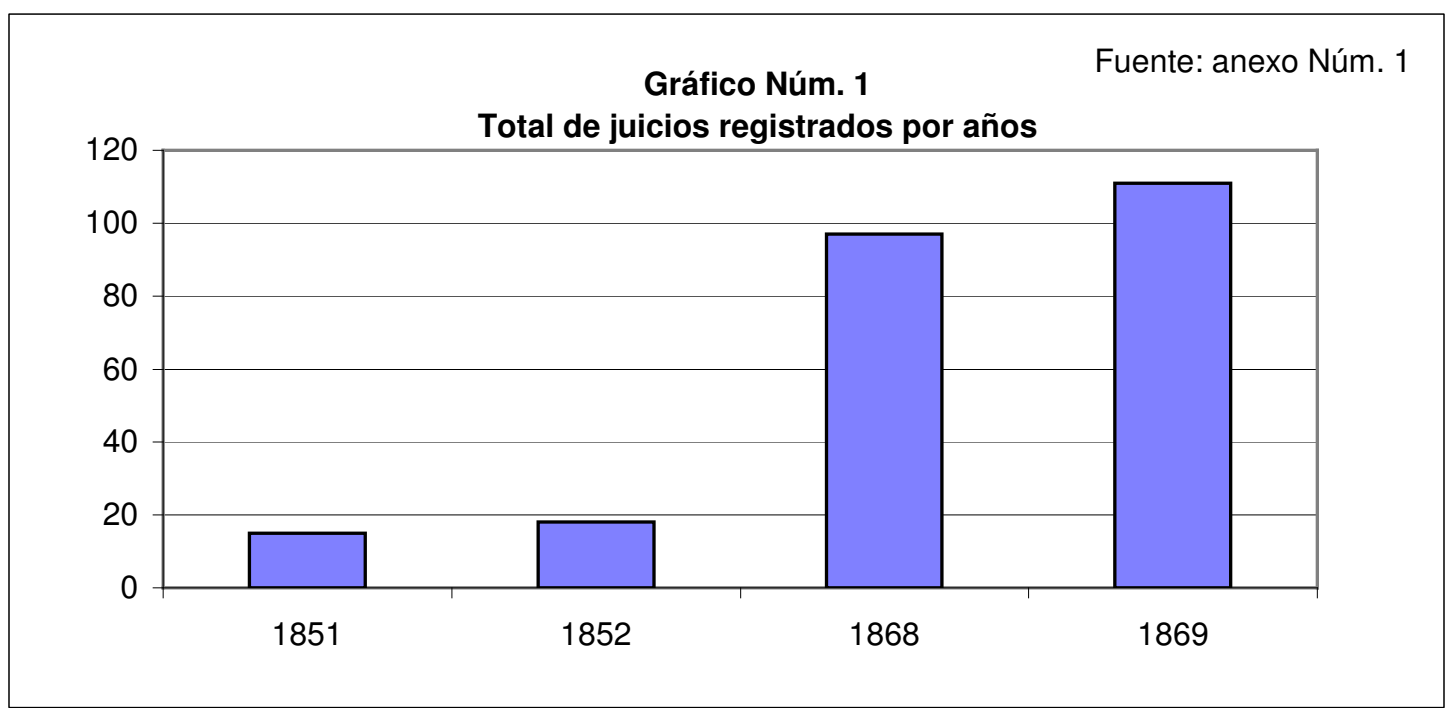


Como se puede notar existe un incremento notorio en las causas civiles después de la segunda mitad del siglo XIX, pasamos de 15 procesos en 1851 a 111 registrados en 1869. Al notar este incremento resulta primordial tratar de averiguar cuál es la causa de éste. Así que en el segundo gráfico trataremos de encontrar alguna explicación al respecto.

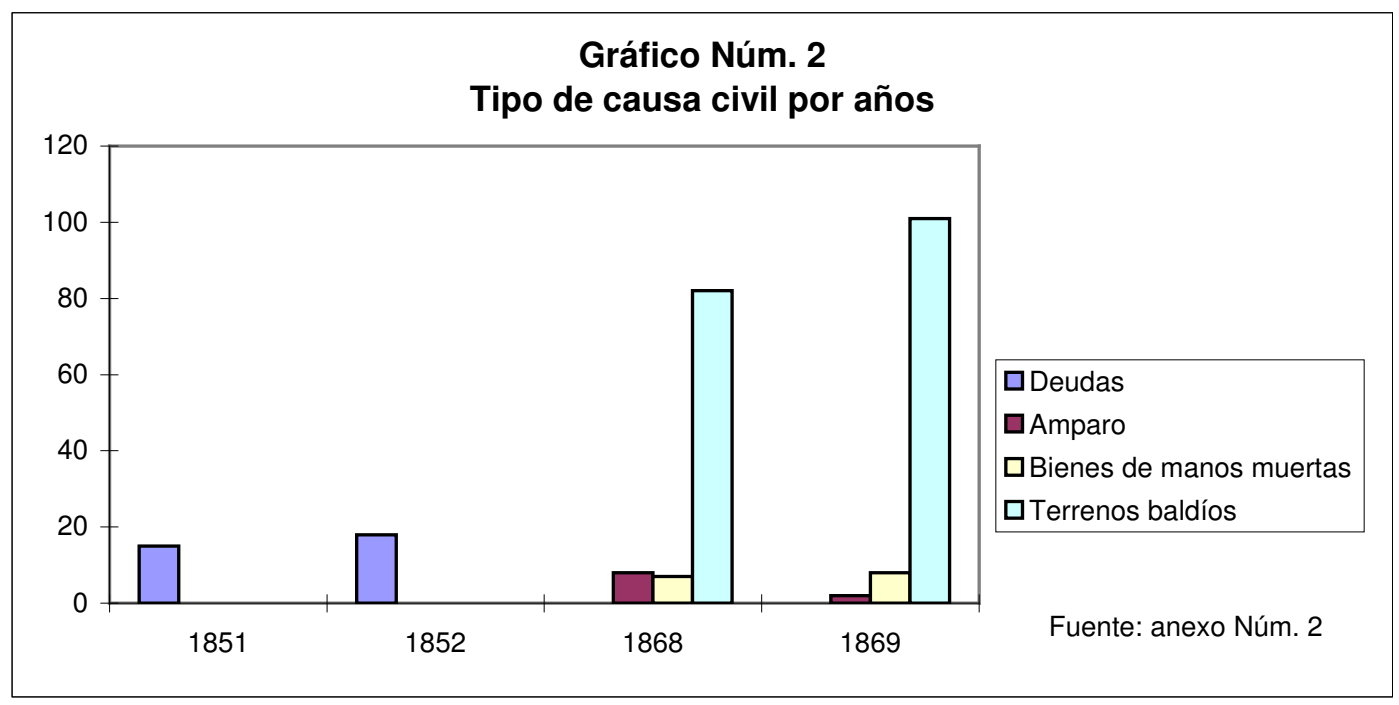

Para facilitar la comparación clasificamos los datos de acuerdo a cuatro categorías básicas, las mismas que organizan el gráfico. Bajo "deudas" agrupamos los juicios entre particulares y el fisco; en el caso de juicios de amparo, estos aparecen sólo en los dos últimos expedientes, seguro por las disposiciones legales al respecto. Para 1868 y 1869 los juicios prácticamente se concentran en aquellos sobre tierras baldías y los bienes de manos muertas. Resulta una incógnita determinar por qué no se mencionan otro tipo de juicios civiles, ni los de hacienda, ni deudas entre particulares, para 1868 y 1869. Tal vez el expediente sólo informó sobre estas categorías por algún cambio de jurisdicción legal o - lo que parece menos probable- los litigios se concentraron en realidad en los tipos mostrados, desapareciendo otro tipo de causas. En todo caso, sea cual fuere la razón, lo importante es la aparición de los juicios por terrenos baldíos en 1868 y 1869.

\footnotetext{
${ }^{8}$ Las fuentes de los gráficos 1 y 2 son los cuadros correspondientes que se presentan al final de este ensayo.
} 
Una afirmación adicional es que la importancia de los juicios por bienes de "manos muertas" es claramente inferior. Tal vez esto pueda ratificar la idea, citada antes, de la importancia relativa del proceso de consolidación de los bienes de las órdenes religiosas por parte de las disposiciones del Estado. Por esta razón nuestro análisis se va a concentrar en el caso de las tierras baldías, pues los datos muestran el impacto de estas leyes antes de 1875 y 1883 , fechas que se mencionan generalmente como importantes de acuerdo a las disposiciones sobre terrenos baldíos. No es que antes de estos últimos años la influencia de las leyes sea superior a lo que ocurre después, sino que propongo que deben ser tomadas en cuenta de manera más consistente. Los datos mostrados por los diversos autores que han trabajado el tema en Chiapas, enfatizan — con absoluta razón - el impacto ocasionado por las leyes del porfiriato. La información que mostramos no intenta corregir tal afirmación, pero sí mostrar que no se debe menospreciar la importancia de las leyes de baldíos de 1863 y, en general, las producidas antes del período de Porfirio Díaz. ${ }^{9}$

Esta afirmación se puede ratificar presentando el año de inicio de los juicios sobre tierras baldías.

\footnotetext{
${ }^{9}$ Para una visión general del proceso véase Pedrero, Gloria. "El proceso de acumulación originaria en el agro chiapaneco, siglo XIX", pp. 31-39, en: Investigaciones recientes en el área maya. San Cristóbal de Las Casas, Sociedad Mexicana de Antropología, 1984.
} 


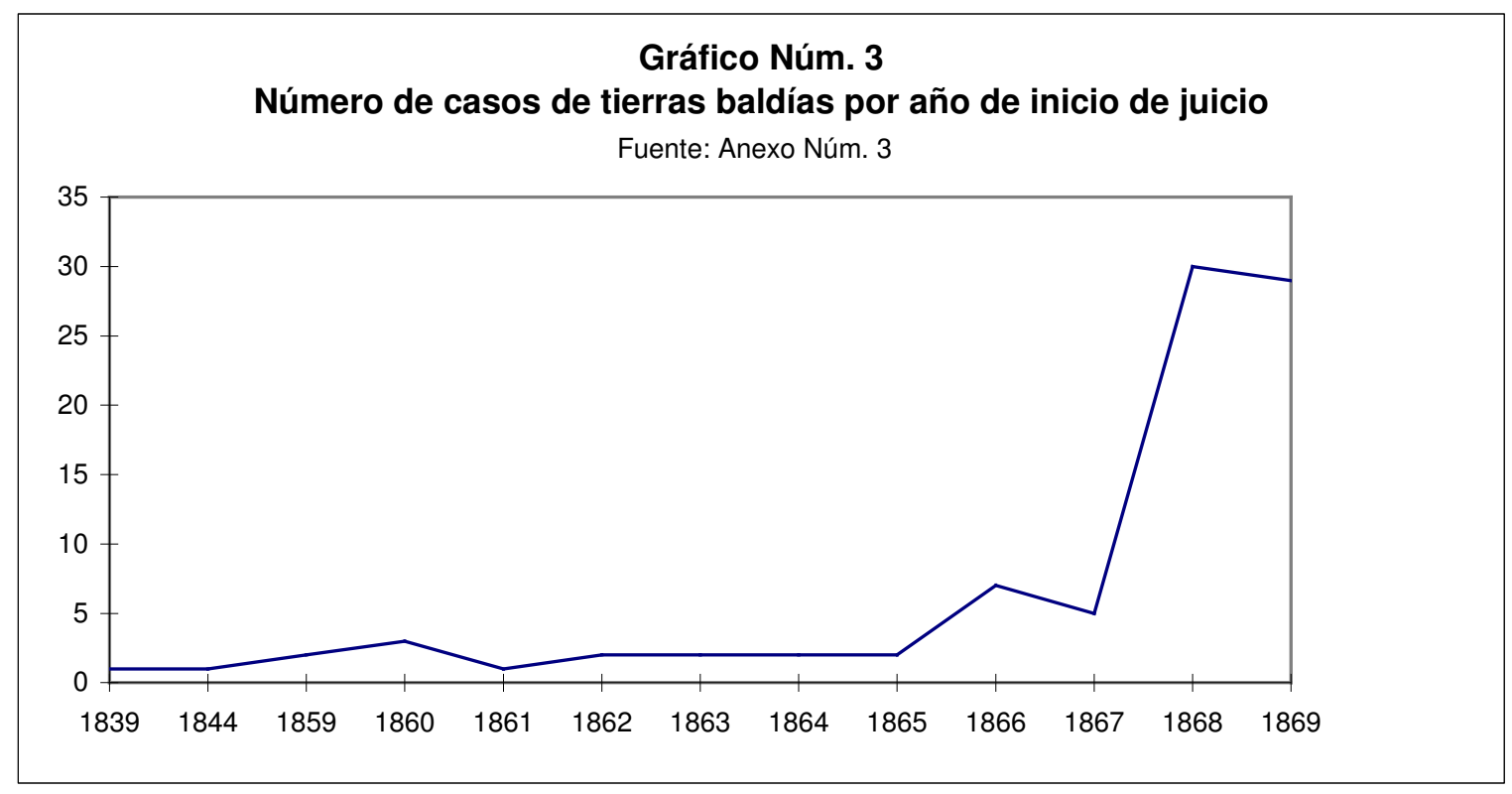

Resulta evidente que en los dos últimos años el número de juicios es numeroso debido —al menos en parte- a que estos se encuentran todavía en curso, de manera que los juicios más antiguos están menos representados porque muchos de ellos ya concluyeron. Si bien esta observación es correcta, aún con todo el incremento de casos es importante de señalar y amerita algún tipo de explicación. Aparentemente después de 1866 las denuncias tienden a incrementarse, coincidiendo con la observación de Mario Ruz respecto a este hecho durante la intervención francesa (1992: 147). En todo caso, estos datos vuelven a llamar la atención sobre el problema de las leyes de baldíos antes del periodo "clásico" del porfiriato. Si la influencia de estas disposiciones resulta importante ¿se podría situar el inicio del crecimiento de las haciendas hacia la década de 1860? Con la información que disponemos no podemos contestar la pregunta, pero los materiales nos sugieren el planteamiento de esta interrogante que puede ser ratificada por otras investigaciones.

En los expedientes de 1868 y 1869, ambos dedicados a un semestre, se consigna la información del estado del juicio. Con este dato podemos ver, más o menos, cuál es el proceso que atraviesan las querellas, las posibles demoras y las causas de su atraso. El siguiente cuadro compara las cifras de ambos semestres. 


\begin{tabular}{|lcccc|}
\hline \multicolumn{5}{c}{ Cuadro Núm. 4} \\
\multicolumn{1}{c|}{ Estado de las causas por años } & & \\
Con el agrimensor & 1868 & $\%$ & 1869 & $\%$ \\
Con el fiscal & 34 & 41 & 65 & 64 \\
Adjudicada & 21 & 26 & 3 & 3 \\
Para adjudicar & 13 & 16 & 0 & 0 \\
En trámite & 8 & 10 & 15 & 15 \\
Abandonada & 5 & 6 & 7 & 7 \\
Sin datos & 0 & 0 & 11 & 11 \\
Total & 1 & 1 & 0 & 0 \\
\hline
\end{tabular}

El juicio incluía, como parte del trámite, las mediciones hechas por el agrimensor para determinar el tamaño del fundo. En algunos casos la demora del juicio era debido a la muerte imprevista del funcionario encargado, lo que hacía alargar el proceso hasta el nombramiento de un reemplazante. Tanto en 1868 como 1869 el mayor número de juicios se encuentra en esta parte del trámite, esperando el deslinde del denuncio para proceder al pago del mismo. Para 1868 el segundo lugar lo constituyen aquellos juicios en manos del fiscal, quien estaba encargado de formar la justicia de la causa. Aparentemente el proceso llevaba sólo algunos meses, pues en 1869 son menos frecuentes. En 1868 el 16\% de las causas llevadas en ese año se habían adjudicado y $10 \%$ estaban listas para lo mismo; mientras en el primer semestre de 1869 no se había entregado ningún terreno, aunque el 15\% se encontraba listo para ser adjudicado, en espera del pago por parte del beneficiario. Resulta enigmático saber por qué en 1869 11\% de las causas resultan abandonadas. Tal vez el denunciante no residía cerca de la zona y llevaba su juicio a la distancia, la otra posibilidad es que probablemente tenía varios juicios o denuncios, lo que dificultaba resolver todos a tiempo. Por último, de manera muy general hemos ubicado a otro tipo de gestión bajo la categoría "en trámite".

Para poder resolver el problema de si existen varias denuncias por persona, antes tuvimos que eliminar la posibilidad de que se repitan de manera natural los nombres, dado que entre 1868 y 1869 es factible que se repita el mismo juicio en ambas listas. Tomando en cuenta dicha posibilidad y revisando el anexo Núm. 1, indicamos algunos nombres que aparecen en varios juicios. 


\begin{tabular}{|c|c|}
\hline \multicolumn{2}{|c|}{$\begin{array}{c}\text { Cuadro № } 5 \\
\text { Personas con más de un juicio } \\
\text { Descripción }\end{array}$} \\
\hline Cleofas Domínguez & $\begin{array}{l}\text { Juicio por asalto y robo (1851-1852) y } \\
\text { terreno para adjudicar en } 1869\end{array}$ \\
\hline Faustino del Pando & Dos terrenos para adjudicar (1869) \\
\hline Lino Argüelles & $\begin{array}{l}\text { Un terreno llamado "Encarnación", } \\
\text { adjudicado en 1868; otro llamado } \\
\text { "Concepción" en manos del fiscal y dos } \\
\text { terrenos baldíos más por adjudicar en } 1869\end{array}$ \\
\hline Luis Córdova & \begin{tabular}{|l|} 
"Llano Taburete" en Comitán (1868) y dos \\
terrenos en manos del agrimensor en 1869
\end{tabular} \\
\hline Martín Gimenes & $\begin{array}{l}\text { Un terreno sin nombre y el "Limón" en } \\
\text { Pichucalco, ambos con el agrimensor en } \\
\text { 1868. Para } 1869 \text { aparece una de sus causas } \\
\text { abandonada }\end{array}$ \\
\hline Nicolás Domínguez & $\begin{array}{l}\text { "La Soledad" en Comitán en manos del } \\
\text { agrimensor en 1868; dos denuncias más en } \\
1869 .\end{array}$ \\
\hline
\end{tabular}

Algunos de estos apellidos resultan importantes para Chiapas. Ignoramos si Cleofas y Nicolás Domínguez pertenecen a la misma familia del gobernador José Pantaleón Domínguez, cuyo círculo es mencionado en la historiografía vinculado al acaparamiento de tierras. García de León menciona varios litigios de tierras de la familia (1985, I: 158); de igual manera Ruz los menciona (1992: 150-151). Lo mismo ocurre con el apellido Argüelles y con Luis Córdova, mencionados también por García de León (Op. cit.); es posible que el proceso haya comenzado algunos años antes de 1868, que es también la fecha inicial de los datos del autor que mencionamos.

Parecen existir muy pocas dudas acerca del crecimiento de las haciendas en la década de 1870. Ruz, citando el estudio de Favre, indica que en 1839 existían 853 "latifundios" que se elevaron a más de tres mil para finales de la década de 1880 (1992: 155). Esto lleva al autor a afirmar que en Chiapas los grandes latifundios "vendrían a crearse exactamente en las regiones boscosas. Comitán, Pichucalco y más tarde el núcleo de la selva Lacandona, serían las zonas más afectadas" (Op. cit.). ${ }^{10}$ Como veremos más adelante, Ruz está acertado en esta afirmación, pues la mayoría de denuncias de tierras baldías se encuentran en esas regiones. Una de las razones que

\footnotetext{
${ }^{10}$ Wasserstrom también afirma el incremento de denuncias en Pichucalco, impulsada por el crecimiento de la demanda en el mercado de azúcar, (Clase y sociedad en el centro de Chiapas. México, FCE, 1989, p.146).
} 
debió influir en las denuncias fue el irrisorio precio que se pagaba por ellas. Para 1863 el gobierno central autorizó la tarifa de 1,65 pesos por hectárea de primera clase, 1,10 por la de segunda y 0,75 cts. por la de tercera. En 1878 se estableció un solo precio de 0,50 cts., tarifa vigente hasta 1880 y que seguramente favoreció de manera enorme el despojo de las tierras comunales (Ruz, 1992: 152). Aunque en apariencia estas disposiciones deberían favorecer la adquisición de tierra por parte de los indígenas para convertirlos en pequeños propietarios, la verdad es que sólo se registra una solicitud de parte de indígenas, además negada en primera instancia, para la compra de terrenos baldíos (Op. cit., 148).

Para mostrar de manera más adecuada el impacto de las denuncias, las veremos a partir del lugar en que se produce el juicio y la ubicación de la finca. ${ }^{11}$ El cuadro siguiente muestra el juzgado de inicio del juicio, no sabemos — por nuestra deficiencia en el conocimiento del entramado legal— hasta que punto coincide el juzgado con la ubicación de la denuncia; además, el dato sólo es mencionado en 1869 y no incluye lugar de la finca ni nombre de ella.

\begin{tabular}{|lc|}
\hline \multicolumn{2}{|c|}{ Cuadro Núm. 6 } \\
Lugar de inicio del juicio (1869) \\
Tuxtla & 63 \\
Tapachula & 3 \\
Soconusco & 1 \\
Pichucalco & 8 \\
Jefatura de Hacienda & 1 \\
Comitán & 12 \\
Chilón & 1 \\
Chiapas & 2 \\
Agencia de Fomento & 5 \\
Sin dato & 5 \\
Total & 101 \\
\hline
\end{tabular}

\footnotetext{
${ }^{11}$ Según García de León una finca "será sinónimo, en Chiapas y América Central, de una unidad de producción comparable (más no idéntica) a la hacienda mexicana. Estas fincas -latifundios y medianas propiedades- estarán dedicadas a la ganadería y al cultivo de maíz, cacao, añil, arroz, chile, tabaco, etc.” (1985, I: 114)
} 
Además de estas observaciones iniciales, el cuadro muestra otra característica. Si bien no sirve para conocer las zonas más solicitadas en las denuncias, nos muestra que la mayoría de los juicios fueron iniciados en el juzgado de Tuxtla (63\%). Los trabajos de García de León y Ruz, los más importantes para nuestro tema, insisten en el enfrentamiento entre un sector conservador, de origen colonial, residente en San Cristóbal y una élite pujante, liberal e interesada en acabar con el predominio de la vieja capital, élite liberal de la cual uno sus núcleos representativos residía en Tuxtla. ${ }^{12}$ Las leyes acerca de la eliminación de los servicios personales y las reminiscencias de tipo colonial, fueron bastante más resistidas en San Cristóbal, mientras eran promovidas desde Tuxtla. Tal vez por esta razón la mayoría de los juicios se llevaban en el juzgado tuxtleco.

\begin{tabular}{|lcc|}
\hline \multicolumn{3}{|c|}{ Cuadro Núm. 7} \\
Ubicación de la finca (1868) \\
Pichucalco & 31 & 38 \\
Comitán & 22 & 27 \\
Palenque & 7 & 9 \\
Soconusco & 7 & 9 \\
Tuxtla & 7 & 9 \\
Chilón & 3 & 4 \\
Chiapa & 2 & 2 \\
Simojovel & 2 & 2 \\
San Cristóbal & 1 & 1 \\
Total & 82 & 100 \\
\hline
\end{tabular}

La ubicación de la finca, registrada en 1868, nos muestra con mayor eficacia la presión sobre las tierras baldías. Es evidente que Pichucalco y Comitán son las zonas más importantes, acumulando entre ellas el $65 \%$ de las denuncias. Como resaltamos líneas atrás, recordando la afirmación de Ruz, en las zonas boscosas, en teoría abandonadas, se concentrarían los esfuerzos para la posesión de tierras baldías. Es posible que estas tierras, como también ya señalé, no se encuentren realmente abandonadas pues la práctica de agricultura rotacional y estacional es muy frecuente en estas zonas. En la realidad este proceso resultaba en simple y llano despojo de tierras utilizadas por las comunidades, en las que no contaban con ningún título legal que avalara su posesión

\footnotetext{
${ }^{12}$ García de León afirma que Comitán entró en pugna con San Cristóbal, incluso se alió con Tuxtla y Chiapa para crear un "cerco liberal" sobre la vieja capital (Ibíd., 142).
} 
Entonces, para concluir esta parte, los datos que mostramos ratifican la idea de un acaparamiento de tierras producida a partir de las denuncias por terrenos baldíos antes que por la Ley Lerdo. Las diversas disposiciones, tanto de elaboración local como nacional, desde 1830 por lo menos, motivaron un proceso de concentración de tierras llevado a cabo con mayor fuerza en las llanuras de Pichucalco y Comitán. Es posible que tenga que ver la demanda de azúcar y la aparición de la ganadería a gran escala en este proceso, pero esto sólo puede ser verificado con un mayor trabajo de archivo. ${ }^{13}$

Quisiéramos terminar indicando algunos de los efectos visibles que trajo este acaparamiento de tierras en Chiapas, reseñados a partir de Ruz y García de León. Los trabajos insisten desde la época colonial en la presencia de "mozos baldíos" como parte de la fuerza de trabajo de las fincas o haciendas. Lo concreto es que la concentración de tierras produjo un crecimiento de este sector de indígenas, quienes "de pronto se encontraron con que sus tierras ya no eran suyas y debían abandonarlas o regalar su trabajo al nuevo dueño si querían seguir habitándolas" (Ruz 1992: 297). Esto produjo migraciones masivas de indígenas, desplazados por el crecimiento de las fincas. En 1868, por ejemplo, 30 familias de indígenas comitecos se quejaron de la hostilidad y violencia con que la familia Figueroa intentaba persuadirlos de que abandonen sus rancherías; hechos como este motivaron la huída de los indígenas hacia zonas más alejadas de los finqueros. Tal vez esto explique por qué en 1862 Comitán, uno de los núcleos más activos en el acaparamiento de tierras, ostentaba el primer puesto de servidumbre en Chiapas ( $O p$. cit., 149 y 306).

Dentro de este proceso de concentración de tierras, motivado por nuevos mercados y necesidades productivas, ocurre, según los autores que seguimos, un aumento de la servidumbre; este fenómeno ha motivado debates teóricos sumamente interesantes. Es decir, la "modernización" de algunas regiones de América Latina, en este caso Chiapas, que ocurrió con la llegada de nuevos capitales y la liberación del mercado de tierras, no produjo el cambio de las relaciones de producción. Al contrario, ocasionó un reforzamiento de las antiguas formas de

\footnotetext{
${ }^{13}$ Ruz menciona una idea similar (1992: 159); García de León sostiene que las necesidades de los hacendados de tierras húmedas para su ganado los llevó a tomar interés en las zonas mencionadas (1992, I: 157).
} 
manejo de la mano de obra. En el caso de Chiapas, por ejemplo, ocasionó el aumento de los "mozos baldíos", producto directo del acaparamiento de tierras. ${ }^{14}$

No podemos dejar de mencionar que este fenómeno también se ha detectado para el caso de las haciendas peruanas, en una zona geográficamente hablando similar a la de Chiapas: pie de montaña, en las cercanías de la selva amazónica vecina del departamento del Cuzco. Como menciona Mörner, los estudios, especialmente de E.J. Hobsbawn, muestran un recrudecimiento de las relaciones serviles a medida que creció el latifundio (1975: 43).

En todo caso me parece muy interesante encontrar bastantes paralelos entre la realidad chiapaneca y las características de los hacendados peruanos, llamados "gamonales". La coexistencia de comunidades, que abastecen de mano de obra a las fincas, enfrentadas a las haciendas, pero también mutuamente dependientes entre sí; más la presencia de "enganchadores", cuyas actividades eran conocidas por el mismo nombre en los Andes y empleaban, además, los mismos métodos, hacen plantear el problema en una dimensión distinta.

Sólo a modo de pensamiento en voz alta me animo a plantear algunas posibilidades. En ambos casos, la sierra del Perú y Chiapas, las comunidades indígenas sobrevivieron bastante bien al mundo colonial. Es decir mantuvieron tierras y cierta autarquía económica. Tal vez por esta razón las frecuentes - y paradójicas - quejas de falta de mano de obra en los Andes se puedan explicar por la imposibilidad de los hacendados, hasta bien entrado el siglo XIX, de generarse un mercado de trabajo dentro de un espacio dominado por las comunidades. Aunque suene muy ortodoxo - y no pienso serlo- tendría que ocurrir una suerte de "despojo inicial", para convertir a los comuneros en peones o "mozos baldíos"15. Esto ocurre con las leyes liberales del siglo XIX en México, mientras en el caso peruano parece que ocurre más bien hacia finales del siglo XIX y principios del siglo XX. En todo caso los paralelos parecen más que sugerentes e invitan al trabajo comparativo.

\footnotetext{
${ }^{14}$ García de León expresa la misma idea (1985, T.I: 165$)$.

${ }^{15}$ Es cierto que hay que tener cuidado con las cifras, no siempre coincide gran número de haciendas con mayoría de población viviendo en ellas. Véase Tortolero, Alejandro. "Hacendados y aperos agrícolas: el caso de la hacienda de San Nicolás del Moral en Chalco, 1880-1916" pp. 318-341, en Miño (1991) y Schenk (1991: 240).
} 


\section{BIBLIOGRAFÍA}

BAZANT, JAN, 1995, "La desamortización de los bienes corporativos en 1856", en A. V. Hernández y M. Chávez, Problemas agrarios y propiedad en México, siglos XVIII y XIX. El Colegio de México, México, pp. 101-120.

DE Vos, JAN, 1995, "Una legislación de graves consecuencias. El acaparamiento de tierras baldías en México, con el pretexto de colonización, 1821-1910”, en A. V. Hernández y M. Chávez, Problemas agrarios y propiedad en México, siglos XVIII y XIX. El Colegio de México, México, pp.227-264.

GonZÁlez NaVArRo, Moisés, 1995, "Las tierras ociosas", en A. V. Hernández y M. Chávez, Problemas agrarios y propiedad en México, siglos XVIII y XIX. El Colegio de México, México, pp. 190-226.

KnOwlton, ROBERT J., 1991, "La individualización de la propiedad corporativa civil en el siglo XIX. Notas sobre Jalisco", en Los pueblos de indios y las comunidades. Lecturas de Historia Mexicana, Vol. 2, El Colegio de México, México, pp. 181-218.

Mejía Fernández, Miguel, 1979, Política agraria en México en el siglo XIX. Siglo XXI, México.

Menegus Bornemann, Margarita, 1995, "Ocoyoacac. Una comunidad agraria en el siglo XIX”, en A. V. Hernández y M. Chávez, Problemas agrarios y propiedad en México, siglos XVIII y XIX. El Colegio de México, México, pp. 144-189.

HERNÁNDEZ CHÁVEZ, A. V. Y M. E. MIÑO GRIJALVA, 1995, Problemas agrarios y propiedad en México, siglos XVIII y XIX. El Colegio de México, México.

MiÑo, Manuel E., (COMP.), 1991 Haciendas, pueblos y comunidades. Los valles de México y Toluca entre 1530 y 1916. CONACULTA, México, 
MÖRner, Magnus, 1975, "La hacienda hispanoamericana: examen de las investigaciones y debates recientes, en E. Florescano (Coord.), Haciendas, Latifundios y Plantaciones en América Latina. Siglo XXI, México, pp. 15-48.

SCHENK, FRANK, 1991, "Jornaleros y hacendados. La distribución de la propiedad de la tierra en el suroeste del Estado de México hacia 1900”, en M. Miño (Comp.), Haciendas, pueblos y comunidades. Los valles de México y Toluca entre 1530 y 1916. CONACULTA, México, pp. 230-269. 